\title{
Warfarin use in hemodialysis patients with atrial fibrillation: decisions based on uncertainty
}

\author{
Salina Juma ${ }^{1}$, Benjamin KA Thomson ${ }^{1,2}$, Charmaine E Lok ${ }^{3}$, Catherine M Clase ${ }^{4,5}$, Peter G Blake ${ }^{1,2}$ \\ and Louise Moist ${ }^{1,2^{*}}$
}

\begin{abstract}
Background: Warfarin prescribing patterns for hemodialysis patients with atrial fibrillation vary widely amongst nephrologists. This may be due to a paucity of guiding evidence, but also due to concerns of increased risks of warfarin use in this population. The literature lacks clarity on the balance of warfarin therapy between prevention of thrombotic strokes and the increased risks of bleeding in hemodialysis patients with atrial fibrillation.

Methods: We performed a survey of Canadian Nephrologists, assessing warfarin prescribing practice, and measured the certainty in making these choices.

Results: Respondents were consistently uncertain about warfarin use for atrial fibrillation. This uncertainty increased with a history of falls or starting hemodialysis, even when a high CHADS2 or CHA2DS2VASc score was present. The majority of respondents agreed that clinical equipoise existed about the use of oral anticoagulation in hemodialysis patients with atrial fibrillation (72.2\%) and that the results of a randomized controlled trial would be relevant to their practice (98.2\%).
\end{abstract}

Conclusions: A randomized controlled trial of warfarin use in hemodialysis patients with atrial fibrillation would clarify the risks and benefits of warfarin use in this population.

Keywords: Warfarin, Anticoagulation, Atrial fibrillation, Hemodialysis, Stroke, CKD, Bleeding

\section{Background}

Warfarin is indicated in the general population for the treatment of venous thromboembolism (VTE) and for prophylaxis of stroke in patients with atrial fibrillation. A recent non-randomized retrospective database analysis confirmed that in patients with non-valvular atrial fibrillation and chronic kidney disease, the use of warfarin or aspirin reduced stroke but increased risk of bleeding [1]. However, in patients on dialysis, warfarin is widely used for nonvalvular atrial fibrillation, though the ratio of risks to benefits remains unclear [2-6]. Warfarin has not been studied in randomized trials in this population, and trials of low-intensity warfarin for the maintenance of access patency showed no benefit $[7,8]$.

\footnotetext{
* Correspondence: Louise.moist@lhsc.on.ca

${ }^{1}$ Kidney Clinical Research Unit, Schulich School of Medicine and Dentistry, Western University, 800 Commissioners Rd E, London, Ontario N6A 5W9, Canada

${ }^{2}$ Division of Nephrology, Department of Medicine, London Health Sciences Center, London, Ontario, Canada

Full list of author information is available at the end of the article
}

The prevalence of atrial fibrillation in patients with endstage kidney disease on hemodialysis is higher than that in the general population, ranging from 7 to $27 \%$ [9-12]. In the United States, the prevalence has tripled from 1992 to 2006. These patients have increased risks for adverse effects from warfarin: major bleeding including hemorrhagic stroke [2,13-15], acceleration of vascular calcification, calcific uremic arteriolopathy and warfarin-induced skin necrosis [16-18]. Patients with severe kidney disease were excluded in the generation and validation of stroke and bleeding risk calculator scores such as CHADS2 and CHA2DS2VASc [19-22], which further raises uncertainty about whether evidence from the general population can be generalized to people on dialysis.

Internationally, warfarin prescribing patterns for hemodialysis patients with atrial fibrillation vary widely, from $2 \%$ in Germany to as high as $37 \%$ in Canada [6]. Since Canadian nephrologists prescribe warfarin frequently in this situation, the objective of our study was to identify the degree of uncertainty and clinical equipoise in these 
decisions for treatment, and to assess support for a randomized trial.

\section{Methods}

\section{National survey of nephrologists}

We developed a survey consisting of six clinicallyrelevant cases, followed by four questions. The cases were designed to include different standardized CHADS2 and CHA2DS2VASc ischemic stroke risk scores, and variable bleeding risk, and were reviewed independently by three nephrologists for clinical validity. The additional questions explicitly evaluated respondents' sense of clinical equipoise, their willingness to enter patients with atrial fibrillation into a randomized controlled trial, and their perceived utility of a randomized control trial of anticoagulation of hemodialysis patients with atrial fibrillation. Respondents were also asked 6 demographic questions, to assess representativeness.

We distributed the survey, using Survey Monkey, to a random sample of members of the Canadian Society of Nephrology. We chose to survey one-third, rather than the whole membership to reduce respondent burden and maximize the response rate. The identical survey was sent three separate times within 6 weeks, at 2 week intervals. Only one response per respondent was permitted.

Fisher's exact test was used to establish $\mathrm{P}$ values between survey response groups. Ethics approval was received by the University of Western Ontario, Health Sciences Research Ethics Board, Research Ethics Board number 17036E).

\section{Results}

A random selection of every third nephrologist member of the Canadian Society of Nephrology was sampled $(\mathrm{n}=90)$, and 56 responded $(62 \%)$, all of whom were responsible for the clinical care of patients on hemodialysis (Figure 1). The majority of respondents practiced in Ontario (54.4\%) or Quebec (15.8\%), in keeping with the large population bases of these two provinces, where $62.0 \%$ of Canada's population resides [23]. Most had more than 11 years experience. Most respondents worked in university affiliated practices $(67.8 \%)$, the remainder in the community, and

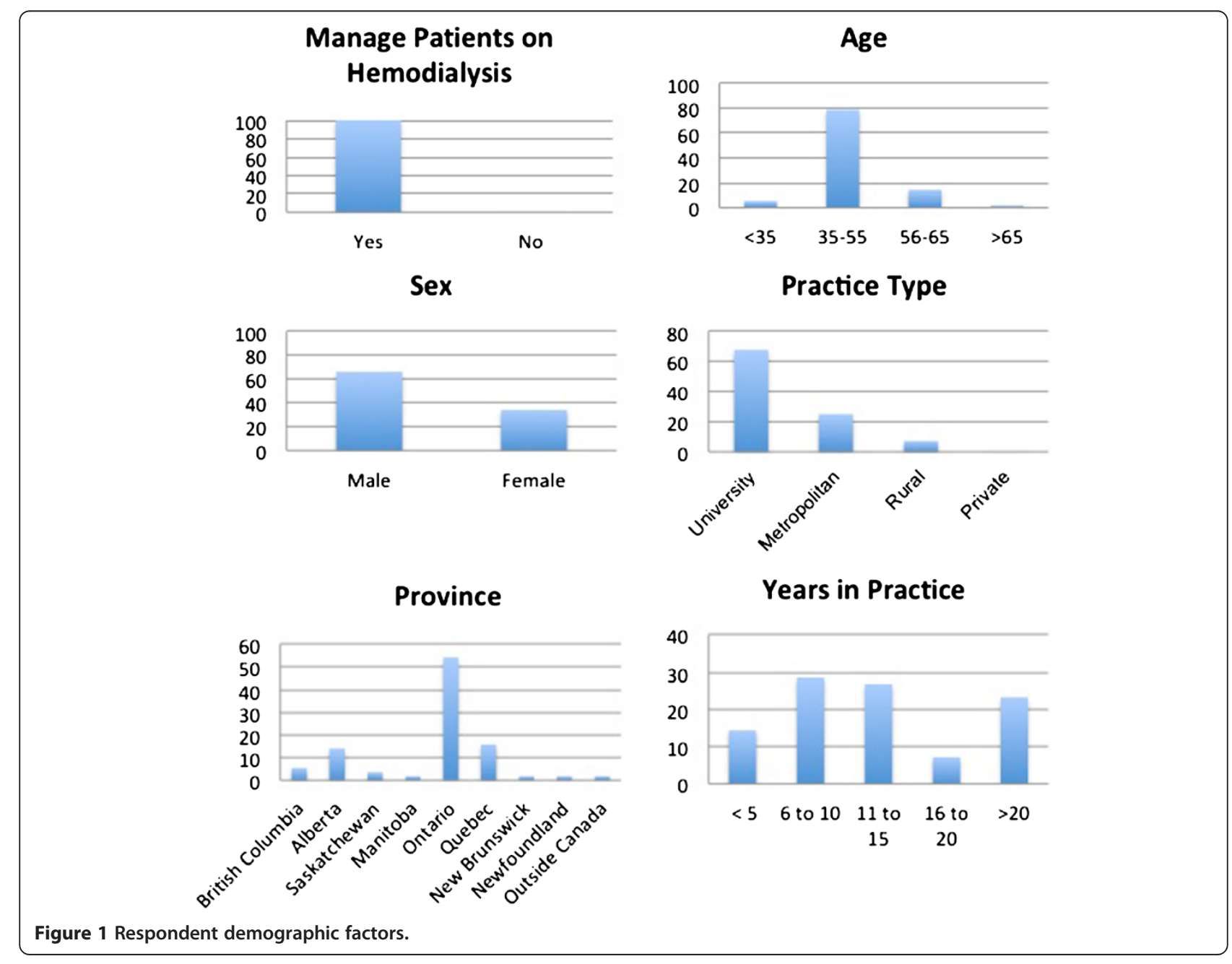


none in private practice, reflecting the Canadian publicly funded health care system.

Responses to each of 6 cases are shown (Table 1). When a patient was placed on hemodialysis, uncertainty to continue warfarin to treat atrial fibrillation increased (from 16 to $36 \%, \mathrm{P}=0.0300$ ), and the likelihood of starting warfarin decreased (from 80 to $50 \%, P=0.0013$ ). Likelihood of warfarin use increased as CHADS2 and CHA2DS2VASc score increased from 2 to 5 (from 50 to $77 \%, \mathrm{P}=0.0057$ ), so long at bleeding risks were absent, and though explicit uncertainty was reduced at the higher scores (36\% falling to $20 \%, P=0.0902)$, significant uncertainty persisted. Half the respondents were uncertain when evaluating a scenario in which the patient was at risk for falls, (Case 4, 48\%), even when there was a high stroke risk $(C H A D S 2=5$, CHA2DS2VASc $=8$ ). Nephrologists were more likely to continue warfarin if there was a history of gastrointestinal bleeding, as opposed to a risk for falls (23\% compared with $48 \%, \mathrm{P}=0.0099$ ), although there was explicit uncertainty (48\%, and $43 \%$, respectively). Very few Nephrologists were likely to prescribe warfarin when a high stroke risk $($ CHADS2 $=5$, CHA2DS2VASc $=8)$ was found with a history of gastrointestinal bleed and risk for falls (3.6\%), while most were unlikely to continue warfarin (68\%); these scenarios highlighted the difference between new prescription and discontinuation of an established therapy (3.6\% prescribing if not taking $\mathrm{v}$ $32 \%$ continuing if already taking, $\mathrm{P}=0.0001$ ). Even in this high-risk scenario, with substantial agreement among nephrologists not to give a new warfarin prescription, explicit uncertainty about the decision was reported by $29 \%$ of nephrologists.

Respondents were asked four questions at the completion of the initial survey about their feeling of clinical equipoise, willingness to enter their own patients into a randomized controlled trial and their feeling that an RCT would be useful in hemodialysis patients with atrial fibrillation (Figure 2). The majority (72\%) mildly or strongly agreed that there was a state of genuine uncertainty within the expert medical community regarding the use of oral anticoagulation in hemodialysis patients with atrial fibrillation. The majority of responders mildly or strongly agreed that they would enroll patients on hemodialysis with atrial fibrillation into a randomized control trial, whether the patient is $(67 \%)$ or is not $(82 \%)$ currently anticoagulated. Finally, the overwhelming majority (98\%) would mildly or strongly agree that the results of a valid and well-conducted RCT on the use of oral anticoagulation in hemodialysis patients with atrial fibrillation would inform their practice.

\section{Discussion}

Our national survey of the standardized cases revealed that using warfarin in a patient on hemodialysis versus a patient with normal renal function increased Nephrologist uncertainty (Figure 1). CHADS2 and CHA2DS2VASc variables unanimously increased the likelihood of prescribing warfarin. However, in the second scenario where the patient had a very high risk for stroke and increased bleeding risks, there was much more variability and Nephrologist uncertainty. Previous gastrointestinal bleed was perceived as lower risk than a patient at risk for falls. As with real patients, Nephrologist ambiguity for warfarin use increased in patients with several comorbidities. There was not always a rationale for physician responses and the responses showed variable physician practices.

We found that asking physicians about their uncertainty directly was most revealing. An overwhelming majority (98.2\%) of Nephrologists agreed that a randomized control trial in this population would inform their practice (Figure 2). Similarly, a majority of $\mathrm{Ne}$ phrologists would enroll their own patients in such a study. Confirming our hypothesis, Nephrologists agreed (72.2\%) that clinical equipoise exists among their community and that a randomized control trial on this topic is needed.

Risk thresholds are a critical component in physician decision-making [24] For example, a decision to anticoagulate a patient with atrial fibrillation must weigh the perceived risks of bleeding and stroke. However, physicians are historically inaccurate in predicting the risks in individual patients in a number of clinical contexts, even when the probabilities of specific outcomes are well defined [25-28]. Furthermore, an accurate assessment of risk requires reliable data on outcomes in the patient group of interest. The establishment of

Table 1 Nephrologist responses, depending on stroke and fall risk, and history of GI bleed

\begin{tabular}{|c|c|c|c|c|c|c|c|c|}
\hline Case & CHADS2 & CHADS-Vasc & Hemodialysis & Gl bleed & $\begin{array}{l}\text { Risk for } \\
\text { falls }\end{array}$ & $\begin{array}{l}\text { Likely to start } \\
\text { warfarin (\%) }\end{array}$ & $\begin{array}{c}\text { Unlikely to } \\
\text { start warfarin (\%) }\end{array}$ & Uncertain (\%) \\
\hline 1 & 2 & 3 & No & No & No & 80.4 & 3.6 & 16.1 \\
\hline 2 & 2 & 3 & Yes & No & No & 50.0 & 14.3 & 35.7 \\
\hline 3 & 5 & 6 & Yes & No & No & 76.7 & 3.6 & 19.6 \\
\hline 4 & 5 & 8 & Yes & No & Yes & 23.2 & 28.6 & 48.2 \\
\hline 5 & 5 & 8 & Yes & Yes & No & 48.2 & 8.9 & 42.9 \\
\hline 6 & 5 & 8 & Yes & Yes & Yes & 3.6 & 67.9 & 28.6 \\
\hline
\end{tabular}


1. Given the current literature available, I feel there is clinical equipoise (a state of genuine uncertainty within the expert medical community) among nephrologists regarding the use of oral anticoagulation in patients with atrial fibrillation on hemodialysis.

2. I would enroll current hemodialysis patients with atrial fibrillation who ARE NOT currently anticoagulated in a randomized trial comparing active anticoagulation versus placebo to evaluate the incidence of major bleeding events and ischemic CVA.

3. I would enroll current patients with atrial fibrillation who ARE currently anticoagulated in a randomized trial comparing active anticoagulation versus placebo to evaluate the incidence of major bleeding events and ischemic CVA.

4. I would use the results of a valid, well-conducted randomized controlled trial on the use of oral anticoagulation in hemodialysis patients with atrial fibrillation to inform my practice.
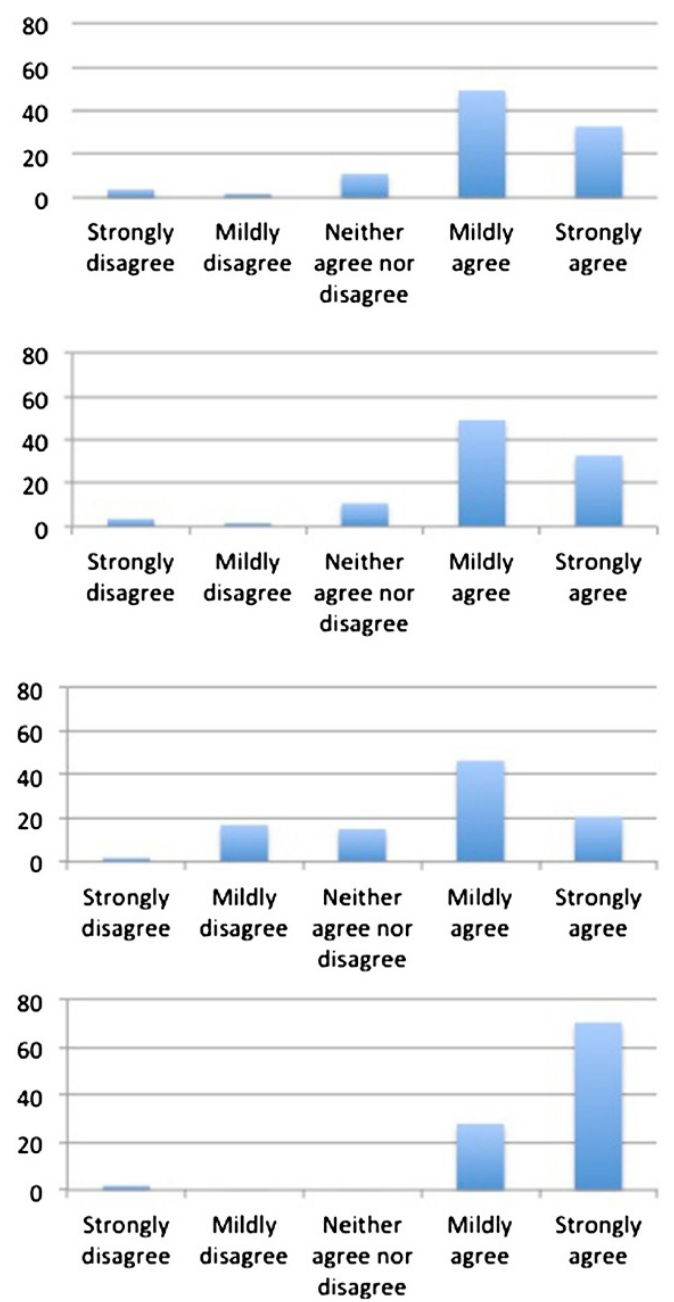

Figure 2 Nephrologist responses to clinical equipoise and willingness to enroll patients in randomized controlled trial. bleeding and stroke outcomes has been challenging and elusive in hemodialysis patients with atrial fibrillation. Certainly this uncertainty can be addressed only with more definitive data on bleeding, stroke and survival, by the conduction of well-designed prospective trials.

The anticoagulation of hemodialysis patients with atrial fibrillation may have unacceptable risks of bleeding [2]. On the contrary, a high proportion of hemodialysis patients have atrial fibrillation, $[3,4]$ and the risk of CVA may or may not be decreased with warfarin use [5,6]. Certainly there would be a substantial health cost savings due to elimination of resources dedicated to monitoring, dosing and prescription of warfarin anticoagulation. However, neither KDOQI nor KDIGO offer recommendations related to chronic anticoagulation of hemodialysis patients. Equipoise reflected in this current study suggests that prospective trials of the two basic strategies (anticoagulation free versus warfarin anticoagulation), would infer the nephrology literature with better clinical practice guidelines.
There would be clear challenges in funding, designing and carrying out a randomized controlled trial on warfarin use in hemodialysis. Since warfarin is inexpensive, readily available and bioequivalent [29], the funding for such a trial would need to come from public, rather than pharmaceutical company funding. Alternatively, a newer non-Vitamin $K$ dependent anticoagulant could be evaluated against warfarin. However, such a study would be challenging in the absence of a placebo patient group, since the question of whether any anticoagulation of any kind is required in this setting would remain unanswered [30]. Furthermore, there is limited experience and safety of newer anticoagulants in hemodialysis patients [31]. Thus, a country with sufficient public funds would need to initiate this rigorously designed, randomized controlled trial.

Designing a randomized controlled trial in this clinical setting would need to overcome ethical challenges. Specifically, randomizing patients to the use of warfarin in hemodialysis patients may be unethical if clinicians 
believe that treatment to be inferior to no anticoagulation. Patients may have strong preferences that limit recruitment and bias outcomes [32]. However, this study confirms that uncertainty is common in Canadian nephrologists, and that willingness to enter patients into a randomized trial is high. Thus, it would not be unethical for Canadian nephrologists to enter their patients into such a trial, since they don't feel that anticoagulation of hemodialysis patients with atrial fibrillation is either inferior or superior. Ultimately, randomized controlled trials are the most rigorous way of determining whether a cause-effect relation exists between treatment and outcome [33], and even despite the above challenges, remains the ideal way to assess the effect of anticoagulation of hemodialysis patients with atrial fibrillation.

There are a number of limitations in our study. Firstly, the survey to the Canadian Society of Nephrologists was limited in that this survey was a novel tool that has not yet been validated. The response rate was limited, however the results still likely reflect the Canadian population of Nephrologists as the demographics of the respondents were fairly representative of our Nephrology population base (Figure 1). Secondly, newer novel oral anticoagulants were not considered in our survey. However, while data on warfarin use in hemodialysis patients is limited, data on novel oral anticoagulant use is nonexistent; thus, warfarin is likely to remain the standard of care in this clinical setting for the foreseeable future. Finally, the generalizability of these results is limited as only Canadians were surveyed. Response bias must also be accounted for when interpreting the results of the survey.

\section{Conclusions}

The results of this study have confirmed that there is limited evidence in the literature to guide decisions on oral anticoagulation in hemodialysis patients with atrial fibrillation. This is reflected in the inconsistent practice among Nephrologists nationally. The paucity of evidence in the literature, and the uncertainty in national practice, lend credence to the performing of a randomized control trial. Indeed, Canadian Nephrologists agree there is clinical equipoise, and are willing to enroll their patients in a well designed randomized control trial.

\section{Abbreviations \\ CHADS2: Congestive heart failure (1) Hypertension (1), Age > 75 (1), Diabetes mellitus (1), Past Stroke or transient ischemic attack (2); CHA2DS2VASc: Congestive Heart Failure (1) Hypertension (1), Age > 75 (2), Diabetes Mellitus (1), Prior Stroke or TIA or thromboembolism (2), Vascular disease (Past myocardial infarction, peripheral artery disease or aortic plaque) (1), Age > 65 (1), Sex category (female gender $=1$ ); CKD: Chronic kidney disease; CSN: Canadian society of nephrology; CVA: Cerebrovascular accident; RCT: Randomized controlled trial.}

\section{Competing interests}

There are no financial competing interests and no non-financial competing interests.

\section{Authors' contributions}

SJ created and distributed the survey, compiled and analyzed the results, then drafted the manuscript. BT analyzed the results, performed statistical analysis, and helped to draft and to modify the manuscript. CEL, CML, PGB participated in the conception and design of the study. LM conceived and designed the study, and helped to draft the manuscript. All authors read and approved the final manuscript.

\section{Acknowledgments}

No additional acknowledgments are required. There are no conflicts of interest identified by any of the authors. No funding source was involved in the collection, analysis or interpretation of the data, nor in the writing of the manuscript or in the decision to submit the manuscript for publication.

\section{Author details}

${ }^{1}$ Kidney Clinical Research Unit, Schulich School of Medicine and Dentistry, Western University, 800 Commissioners Rd E, London, Ontario N6A 5W9, Canada. ${ }^{2}$ Division of Nephrology, Department of Medicine, London Health Sciences Center, London, Ontario, Canada. ${ }^{3}$ Division of Nephrology, Department of Medicine, Toronto General Hospital, University of Toronto, Toronto, Ontario, Canada. ${ }^{4}$ Department of Medicine, McMaster University, Hamilton, Ontario, Canada. ${ }^{5}$ Department of Clinical Epidemiology and Statistics, McMaster University, Hamilton, Ontario, Canada.

Received: 2 August 2012 Accepted: 30 July 2013

Published: 13 August 2013

\section{References}

1. Olesen J, Lip G, Kamper A, Hommel K, Kober L, Lane D, Lindharden J, Gislason G, Torp-Pederson C: Stroke and bleeding in atrial fibrillation with chronic kidney disease. N Engl J Med 2012, 367:625-635.

2. Elliott M, Zimmerman D, Holden R: Warfarin anticoagulation in hemodialysis patients: a systematic review of bleeding rates. Am J Kidney Dis 2007, 50:433-440.

3. Genovesi S, Vincenti A, Rossi E, Pogliani D, Acquistapace I, Stella A, Valsecchi MG: Atrial fibrillation and morbidity and mortality in a cohort of longterm hemodialysis patients. Am J Kidney Dis 2008, 51(2):255-262.

4. Wiesholzer M, Harm F, Tomasec G, Barbieri G, Putz D, Balcke P: Incidence of stroke among chronic hemodialysis patients with nonrheumatic atrial fibrillation. Am J Nephrol 2001, 21(1):35-39.

5. Chan KE, Lazarus JM, Thadhani R, Hakim RM: Warfarin use associates with increased risk for stroke in hemodialysis patients with atrial fibrillation. J Am Soc Nephrol 2009, 20(10):2223-2233.

6. Wizemann V, Tong L, Satayathum S, Disney A, Akiba T, Fissell RB, Kerr PG Young EW, Robinson BM: Atrial fibrillation in hemodialysis patientsL clinical features and associations with anticoagulant therapy. Kidney Int 2010, 77(12):1098-1106.

7. Mokrzycki MH, Jean-Jerome K, Rush H, Zdunek MP, Rosenberg SO: A randomized trial of minidose warfarin for the prevention of late malfunction in tunneled, cuffed hemodialysis catheters. Kidney Int 2001, 59:1935-1942

8. Crowther MA, Clase CM, Margetts PJ, Julian J, Lambert K, Sneath D, Nagai R, Wilson S, Ingram AJ: Low-intensity warfarin is ineffective for the prevention of PTFE graft failure in patients on hemodialysis: A randomized controlled trial. J Am Soc Nephrol 2002, 13:2331-2337.

9. Abe S, Yoshizawa M, Nakanishi N, Yazawa T, Yokota K, Honda M, Sloman G: Electrocardiographic abnormalities in patients receiving hemodialysis. Am Heart J 1996, 131:1137-1144.

10. Fabbian F, Catalano C, Lambertini D, Tarroni G, Bordin V, Squerzanti R, Gilli P, Di Landro D, Cavagna R: Clinical characteristics associated to atrial fibrillation in chronic hemodialysis patients. Clin Nephrol 2000, 54:234-239.

11. Vazquez E, Sanchez-Perales C, Borrego F, Garcia-Cortes MJ, Lozana C, Guzman M, Gil JM, Borrego MJ, Perez V: Influence of atrial fibrillation on the morbido-mortality of patients on hemodialysis. Am Heart J 2000, 140:886-890.

12. Genovesi S, Pogliani D, Faini A, Valsecchi MG, Riva A, Stefnai F, Acquistapace I, Stella A, Bonforte G, DeVecchi A, DeCristofaro V, Buccianti G, Vincenti A: Prevalence of atrial fibrillation and associated factors in a population of long-term hemodialysis patients. Am J Kidney Dis 2005, 46:897-902. 
13. Seliger S, Gillen D, Longstreth WJ, Kestenbaum B, Stehman-Breen C: Elevated risk of stroke among patients with end-stage renal disease. Kidney Int 2003, 64:603-309.

14. Kawamura M, Fijimoto S, Hisanaga S, Yamamoto Y, Eto T: Incidence, outcome, and risk factors of cerebrovascular events in patients undergoing maintenance hemodialysis. Am J Kidney Dis 1998, 31:991-996.

15. Holden R, Harman G, Wang M, Holland D, Day A: Major bleeding in hemodialysis patients. Clin J Am Soc Nephrol 2008, 3:105-110.

16. Hayashi M, Takamatsu I, Kanno Y, Yoshida T, Abe T, Sato Y, for the Japanese Calciphylaxis Study Group: A case-control study of calciphylaxis in Japanese end-stage renal disease patients. Nephrol Dial Transplant 2012, 27(4):1580-1584

17. Rockx MA, Sood MM: A necrotic skin lesion in a dialysis patient after the initiation of warfarin therapy: a difficult diagnosis. J Thromb Thrombolysis 2010, 29(1):130-133

18. Soundararajan R, Leehey DJ, Yu AW, Ing TS, Miller JB: Skin necrosis and protein $\mathrm{C}$ deficiency associated with vitamin $\mathrm{K}$ depletion in a patient with renal failure. Am J Med 1992, 93(4):467-470.

19. Gage BF, Waterman AD, Shannon W, Boechler M, Rich MW, Radford MJ: Validation of clinical classification schemes for predicting stroke: results from the National Registry of Atrial Fibrillation. JAMA 2001, 285:2864-2870

20. Fuster V, Ryden LE, Cannom DS, Crijns HJ, Curtis AB, Ellenbogen KA, Halperin JL, Kay GN, Le Huezey JY, Lowe JE, Olsson SB, Prystowsky EN, Tamargo JL, Wann LS: 2011 ACCF/AHA/HRS focused updates incorporated into the ACC/AHA/ESC 2006 guidelines for the management of patients with atrial fibrillation: a report of the American College of Cardiology Foundation/American Heart Association Task Force on Practice Guidelines developed in partnership with the European Society of Cardiology and in collaboration with the European Heart Rhythm Association and the Heart Rhythm Society. J Am Coll Cardiol 2011, 57:e101-e198.

21. Crandall MA, Horne BD, Day JD, Anderson JL, Muhlestein JB, Crandall BG, Weiss JP, Osborne JS, Lappe DL, Bunch TJ: Atrial fibrillation significantly increases total mortality and stroke risk beyond that conveyed by the CHADS2 risk factors. Pacing Clin Electrophysiol 2009, 32:981-986.

22. Lip GY, Nieuwlaat R, Pisters R, Lane DA, Crijns HJ: Refining clinical risk stratification for predicting stroke and thromboembolism in atrial fibrillation using a novel risk factor-based approach: the euro heart survey on atrial fibrillation. Chest 2010, 137:263-272.

23. Statistics Canada: 2011 Population Census [Data file]; 2011. http://www. statcan.gc.ca/tables-tableaux/sum-som/l01/cst01/demo02a-eng.htm.

24. Boland MV, Lehmann HP: A new method for determining physician decision thresholds using empiric, uncertain recommendations. BMC Med Inform Decis Mak 2010, 10:20

25. Poses RM, Cebul RD, Collins M, Fager SS: The accuracy of experienced physicians' probability estimates for patients with sore throats. Implications for decision making. JAMA 1985, 254(7):925-929.

26. Dolan JG, Bordley DR, Mushlin Al: An evaluation of clinicians' subjective prior probability estimates. Med Decis Making 1986, 6(4):216-223.

27. Bobbio M, Fubini A, Detrano R, Shandling AH, Ellestad MH, Clark J, Brezden O, Abecia A, Martinez-Caro D: Diagnostic accuracy of predicting coronary artery disease related to patients' characteristics. J Clin Epidemiol 1994, 47(4):389-395.

28. Chatellier G, Blinowska A, Menard J, Degoulet P: Do Physicians estimate reliably the cardiovascular risk of hypertensive patients. Medinfo 1995 8(Part 2):876-879.

29. Dentali F, Donadini MP, Clark N, Crowther MA, Garcia D, Hylek E, Witt DM, Ageno W, Warfarin Associated Research Projects and Other Endeavors (WARPED) Consortium: Brand name versus generic warfarin: a systematic review of the literature. Pharmacotherapy 2011, 31(4):386-393.

30. Clase CM, Holden RM, Sood MM, Rigatto C, Moist LM, Thomson BK, Mann JF, Zimmerman DL: Should patients with advanced chronic kidney disease and atrial fibrillation receive chronic anticoagulation? Nephrol Dial Transpl 2012, 27(10):3719-3724.
31. Poulsen BK, Grove EL, Husted SE: New oral anticoagulants: a review of the literature with particular emphasis on patients with impaired renal function. Drugs 2012, 72(13):1739-1753.

32. Altman DG: Better reporting of randomized controlled trials: The CONSORT statement. Br Med J 1996, 313:370-371.

33. Sibbald B, Roland M: Understanding controlled trials: Why are randomized controlled trials important? Br Med J 1998, 316:201.

doi:10.1186/1471-2369-14-174

Cite this article as: Juma et al:: Warfarin use in hemodialysis patients with atrial fibrillation: decisions based on uncertainty. BMC Nephrology $201314: 174$

\section{Submit your next manuscript to BioMed Central and take full advantage of:}

- Convenient online submission

- Thorough peer review

- No space constraints or color figure charges

- Immediate publication on acceptance

- Inclusion in PubMed, CAS, Scopus and Google Scholar

- Research which is freely available for redistribution

Submit your manuscript at www.biomedcentral.com/submit
C BioMed Central 